\title{
Functional implications of root cortical senescence for soil resource capture
}

\author{
Hannah M. Schneider • Jonathan P. Lynch
}

Received: 27 September 2017 / Accepted: 11 December 2017 / Published online: 19 December 2017

(C) Springer International Publishing AG, part of Springer Nature 2017

\begin{abstract}
Background Root phenes play a primary role in plant adaptation to edaphic stress. Understanding the functional implications of root phenotypes will enable the development of crop varieties with improved soil resource acquisition. Root cortical senescence (RCS) is a type of programmed cell death in cortical cells of several Poaceae species. Until recently there has been very little attention to the functional implications of RCS for water and nutrient capture.

Scope We explore the functional implications of RCS as an adaptive trait for water and nutrient acquisition. The present review summarizes evidence from our own studies and other published work, and provides novel insights into the fitness landscape of RCS. Progress has recently been achieved in understanding the development and physiological implications of RCS. We propose that RCS is a useful trait for water and nutrient acquisition, particularly in edaphic stress conditions.

Conclusions Further research is needed to understand the utility and tradeoffs of RCS in the context of specific environments, management practices, and phenotypic backgrounds. The utility of RCS for improved plant performance under edaphic stress merits investigation in the field. RCS may be a useful breeding target for
\end{abstract}

Responsible Editor: Philippe Hinsinger.

H. M. Schneider · J. P. Lynch $(\bowtie)$

Department of Plant Science, The Pennsylvania State University, University Park, PA 16802, USA

e-mail:.jp14@psu.edu improved soil resource capture in several major crop species including wheat, barley, and triticale.

Keywords Ethylene $\cdot$ Phene $\cdot$ Programmed cell death . Radial transport $\cdot$ Root $\cdot$ Root cortical senescence
Abbreviations
PCD Programmed cell death
RCS Root cortical senescence
RCA Root cortical aerenchyma

\section{The importance of root phenes for global agriculture}

Drought and nutrient stress are primary global constraints to agricultural productivity (Tilman et al. 2002; Lynch 2007; Lobell et al. 2014). Climate change is projected to intensify the importance of crop water stress as a result of altered precipitation patterns, and soil degradation (Tebaldi and Lobell 2008; Brisson et al. 2010). In many areas of the world, fertilizer use is low and prospects for increased irrigation are limited as most smallholder farms have limited access to fertilizer (Azeez et al. 2005) and freshwater availability is decreasing both in quantity (e.g. aquifer depletion) and quality (e.g. salinization) (Tebaldi and Lobell 2008). In developed countries, intensive fertilization is a primary energy, economic, and environmental cost of agricultural production (Woods et al. 2010). Understanding phenes ('phene' is to 'phenotype' as 'gene' is to 'genotype') (Lynch 2011; Pieruschka and Poorter 2012; York 
et al. 2013) that enhance plant performance in drought and nutrient-limited conditions is important in increasing the productivity, resilience, and sustainability of global agriculture. Root phenes are obviously of primary importance in soil resource capture, but the fitness impacts and genetic control of root phenes are not well understood. Understanding the fitness landscape (Lynch and Brown 2012) of root phenes may enable the development of new crop cultivars with improved root phenotypes for soil resource acquisition.

Root architectural phenes regulate the placement of roots in distinct soil domains, and subsequently affect the acquisition of mobile and immobile nutrients. The acquisition of mobile resources, including nitrate and water, is enhanced by root phenotypes that increase the exploration of deep soil domains. Over the growing season, mobile resources are available in deep soil domains due to depletion by root uptake in the topsoil as loss to leaching in the case of nitrate and direct evaporation in the case of water (Manschadi et al. 2006; Lynch 2013; Trachsel et al. 2013; Lynch and Wojciechowski 2015). However, immobile resources, including potassium and phosphorus, typically have the greatest availability in shallow soil domains (Ho et al. 2005; Zhu et al. 2005; Lynch 2011; Lynch 2013).

Root anatomical phenes also have important roles in soil resource capture. Several root anatomical phenes influence the metabolic cost of soil exploration by changing the proportion of respiring and non-respiring root tissue and affecting the nutrient and carbon cost of tissue construction and maintenance. Plants that are able to access soil resources at a reduced metabolic cost will have superior productivity because more metabolic resources will be available for further resource acquisition, growth, and reproduction (Lynch 2013; Lynch 2015). Cortical phene states that reduce living cortical tissue reduce root respiration and nutrient content, thereby permitting greater resource allocation to other plant functions including growth and reproduction (Lynch and Ho 2005; Lynch 2015). For example, the development of root cortical aerenchyma (RCA) in maize improves plant performance in environments with suboptimal nutrient and water availability (Zhu et al. 2010; Postma and Lynch 2011a, b; Jaramillo et al. 2013; Saengwilai et al. 2014; Chimungu et al. 2015), an effect which modeling studies show can be attributed to a reduction in root metabolic costs (Postma et al. 2014; Dathe et al. 2016). Fewer cell files or greater cell size in the root cortex of maize substantially reduces root respiration and improves soil exploration and water acquisition in conditions of suboptimal water availability (Chimungu et al. 2014a, 2014b). Recently, root cortical senescence (RCS), a phene anatomically distinct from RCA, has also been identified as a root phene that reduces root metabolic costs, thereby improving plant growth under suboptimal nutrient availability. Here, we do not provide a comprehensive review of RCS but rather highlight key studies on RCS and identify research opportunities to enable further understanding of the developmental, physiological, and genetic mechanisms controlling RCS.

\section{Root cortical senescence}

RCS is a type of programmed cell death (PCD) in root cortical cells (Fig. 1) in Poaceae and forms in many species including triticale (Triticosecale) (Liljeroth et al. 2001; Liljeroth and Bryngelsson 2002), barley (Hordeum vulgare L.) (Deacon and Henry 1980; Liljeroth and Bryngelsson 2002), rye (Secale cereal) (Liljeroth 1995), oats (Avena sativa) (Liljeroth 1995), Kentucky bluegrass (Poa pratensis) (Smiley and Giblin 1986), perennial ryegrass (Lolium perenne) (Jupp and Newman 1987), blue grama grass (Bouteloua gracilis) (Beckel 1956), and wheat (Triticum aestivum) (Deacon and Henry 1980; Lascaris and Deacon 1991a; Liljeroth and Bryngelsson 2002). RCS was first observed by Henrici (1929) in South African xeric grasses and was first quantified by Holden (1975) using nuclear staining methods. In three-week-old root segments of wheat, the majority of root cortical cells were anucleate, although the roots still appeared white and healthy (Holden 1975; Holden 1976). Holden's findings were later confirmed (Deacon and Henry 1978; Henry and Deacon 1981; Kirk and Deacon 1986) and the phenomenon was termed 'non-pathogenic root cortex death' (Deacon and Henry 1978) and more recently referred to as RCS (Robinson 1990; Liljeroth 1995; Schneider et al. 2017a).

\section{Regulation of RCS development}

The spatiotemporal development of RCS has been well characterized. Cytoplasmic and nuclear stains have demonstrated that cortical cells of seminal and nodal roots in several Poaceae species can senesce very early in plant growth, leaving the tips of the root axes and 


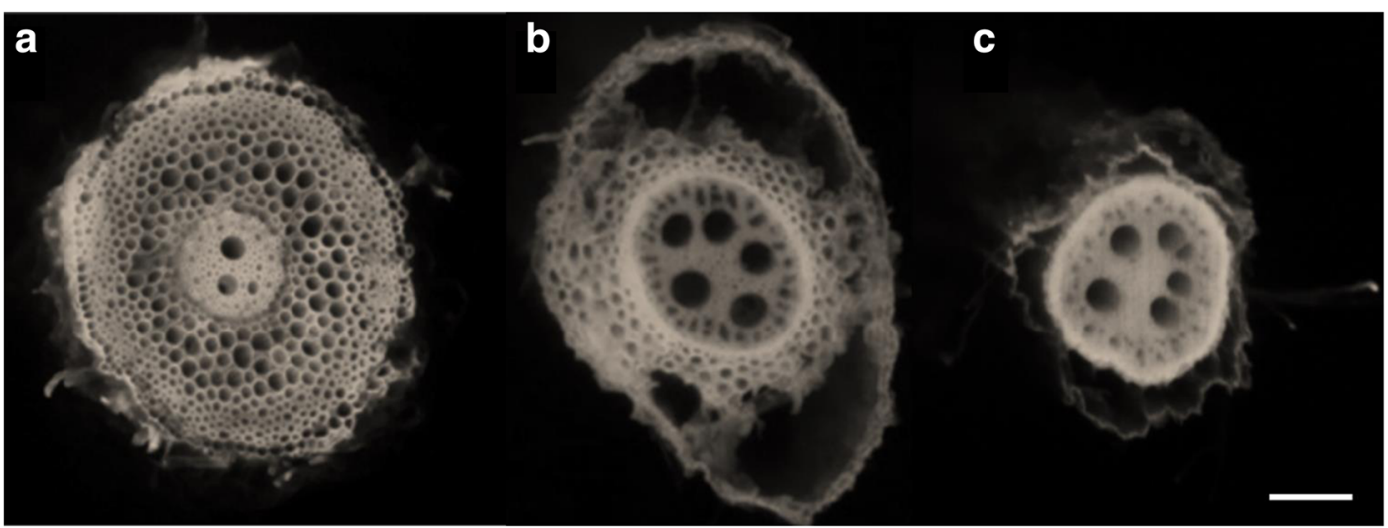

Fig. 1 Progression of RCS in a nodal root of barley at 35 days after germination. Root transverse cross sections at A) $4 \mathrm{~cm}$ behind the root apex B) $10 \mathrm{~cm}$ behind the root apex c) $24 \mathrm{~cm}$ behind the

lateral roots to continue to grow (Holden 1975; Henry and Deacon 1981; Deacon and Lewis 1982; Deacon and Mitchell 1985; Schneider et al. 2017a). RCS begins in outer cortical cell files, directly behind the zone of anucleate root hairs, and progresses inwards and basipetally resulting in anucleate cortical cells. Eventually nearly all cortical cell files in nodal and seminal roots from approximately two $\mathrm{cm}$ behind the root apex to approximately three $\mathrm{cm}$ from the root-shoot junction will become anucleate (Henry and Deacon 1981; Deacon and Lewis 1982; Kirk and Deacon 1986; Wenzel and McCully 1991; Schneider et al. 2017a) (Figs. 1 and 2). The development of RCS is reduced or delayed in the axial root around the base of lateral roots, an effect that extends to approximately $800 \mu \mathrm{m}$ around the base of each lateral and most pronounced on the side of the axis where the lateral emerges (Van Vuurde and Schippers 1980; Henry and Deacon 1981). However, the delayed RCS effect caused by lateral roots is temporary as with increasing distance behind the apex, the number of anucleate cell files around the base of lateral roots progressively increases (Henry and Deacon 1981). In addition, lateral roots themselves do not form RCS (Henry and Deacon 1981; Schneider et al. 2017b). RCS is complex and dependent on plant species, growth medium, and genetic and environmental cues (Liljeroth 1995; Schneider et al. 2017a).

RCS progresses fastest in the seminal roots of wheat followed by triticale, barley, rye, and oats respectively (Holden 1976; Deacon and Henry 1980; Henry and Deacon 1981; Deacon and Mitchell 1985; Yeates and Parker 1985; Kirk and Deacon 1986; Liljeroth 1995). In 15-day-old segments of wheat roots grown in soil, root apex. RCS begins in the outer cortical cell files and progresses inwards (B) and eventually all cortical cells will senescence only leaving the stele viable $(\mathrm{C})$. Scale bar $=100 \mu \mathrm{m}$

between 80 and $90 \%$ of the root cortical cells were anucleate, in contrast to between 20 and $35 \%$ anucleate cortical cells in barley and rye (Liljeroth 1995). Several studies claim the identification of RCS in dicotyledonous species including in peanut (Arachis hypogaea) (Griffin et al. 1975) and rape (Brassica napus L.) (MacLeod et al. 1986). However, dicotyledonous species have fundamentally different root growth compared to monocotyledonous species. Roots of dicot plants undergo secondary growth, typically destroying the cortex and rupturing the epidermis in a different pattern than RCS. Evidence suggests that dicotyledonous species do not undergo RCS and it may be a phenomenon primarily in Poaceae species (Krauss and Deacon 1994; Bingham 2007).

The development of RCS is also influenced by cultivar. Differences in the rate of RCS were observed among four perennial ryegrass varieties (Kirk and Deacon 1986), six spring wheat varieties (Henry and Deacon 1981), and fourteen different barley varieties (Liljeroth 1995). Landraces and modern varieties of barley have different rates of RCS progression and landraces have a faster progression of RCS compared to modern varieties (Schneider et al. 2017a). Although genetic variation for RCS has been characterized, the genetic mechanisms controlling RCS are poorly understood. RCS is influenced by the substitution of the chromosome pairs $5 \mathrm{~B}$ or $5 \mathrm{D}$ between commercial varieties of spring wheat (Deacon and Lewis 1982). Further insights of the genetic mechanisms controlling RCS would enable the further characterization of this trait and perhaps the generation of isogenic lines to further understand its adaptive significance. The presence of 
Fig. 2 Progression of RCS in nodal and seminal roots in a modern cultivar. The same pattern of programmed cell death occurs in seminal and nodal roots. RCS does not develop in lateral roots. Root longitudinal figure modified from Sparks (2017)

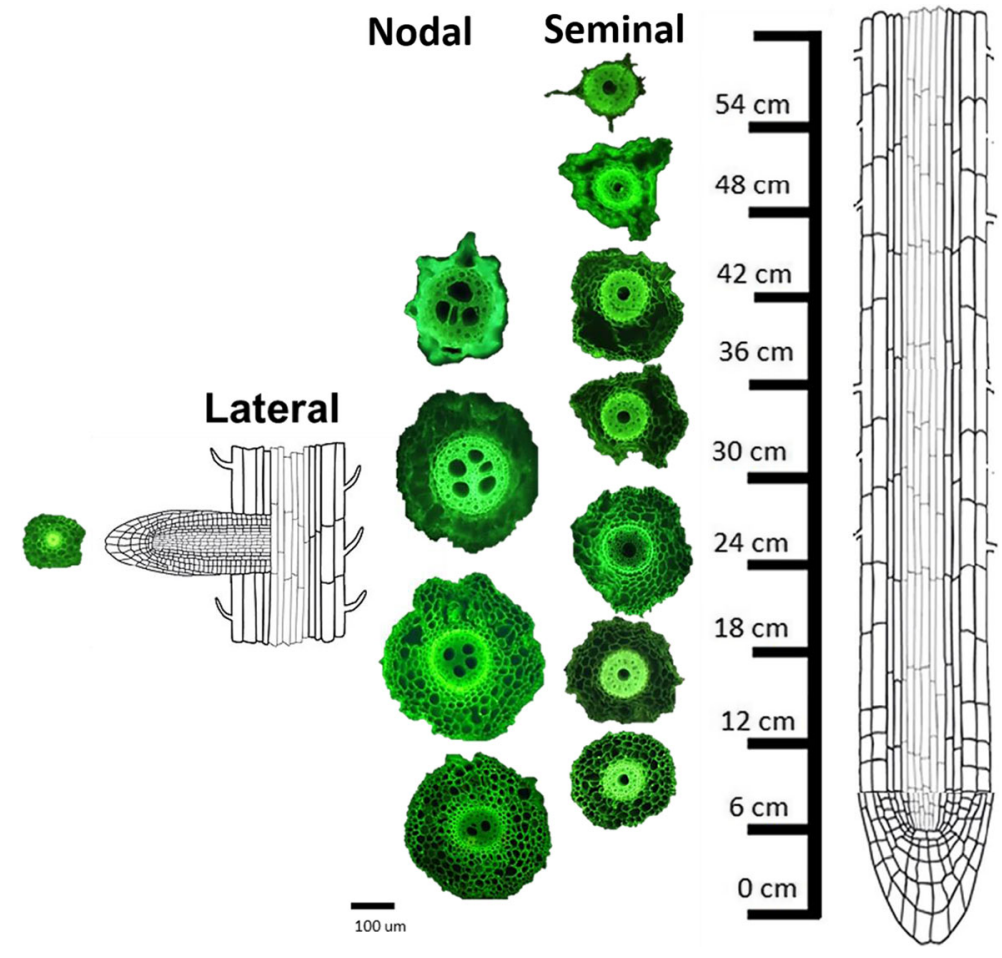

genetic variation suggests that RCS has variable effects on fitness in different environments.

RCS develops at the same rate in sterile and nonsterile conditions and is not enhanced by the presence of soil microorganisms or fungi (Henry and Deacon 1981; Lewis and Deacon 1982). However, RCS influences the size (Van Vuurde and Schippers 1980) and types (Deacon and Lewis 1982; Deacon and Lewis 1986) of rhizosphere bacterial and fungal populations, and regions of spring wheat roots that develop RCS support larger rhizosphere populations compared to regions with no RCS (Van Vuurde and Schippers 1980; Deacon and Lewis 1982; Deacon and Lewis 1986). However, the longevity of cortical cells has important implications in determining the potential resistance of the cortex to a number of diseases (Yeates and Parker 1985). RCS is typically followed by the growth of fungi and bacteria in living and dead root tissues (Old and Nicolson 1975). After RCS, the root cortex has reduced capacity for defense including the formation of lignotubers or cell wall thickening in response to fungal hyphae (Holden 1976). In addition, nutrients leaked from senescing cells may be used by colonizing microorganisms. Patterns of fungal invasion closely match cortical cell death patterns of RCS (Deacon 1980; Deacon and Henry 1980; Deacon and Lewis
1986) and a strong positive correlation was observed between the rate of RCS and fungal biomass in the root (Liueroth et al. 1996). RCS is not induced by fungi or bacteria, rather the nutrients from senescing cells and reduced cortical defense responses enable the growth of fungi and bacteria in the cortex and rhizosphere.

Mechanisms to manipulate RCS would enable further studies of the functional implications of RCS. Several factors control the rate of RCS including ethylene and suboptimal nutrient availability (Schneider et al. $2017 \mathrm{a}, \mathrm{c})$. Ethylene has pronounced effects on the rate of RCS. Cobalt(II), a known inhibitor of ethylene synthesis (Yu and Yang 1979), reduced the rate of RCS in root segments on agar media (Lascaris and Deacon 1991b). Seminal and nodal roots treated with exogenous ethylene had $35 \%$ and $46 \%$ more RCS, respectively compared to the control (aeration) treatment. The accelerated progression of RCS with root-zone ethylene was completely reversed with an ethylene action inhibitor. However, an ethylene action inhibitor did not reduce RCS compared to the control (Schneider et al. 2017c). This indicates that ethylene may play a role in regulating the rate of RCS, but is not required for the development of RCS.

Nutrient availability also regulates the rate of RCS development. Nutrient deficiencies do not affect the 
pattern or where RCS begins relative to the root apex, rather the rate of RCS development. RCS was accelerated by suboptimal availability of nitrogen (Lascaris and Deacon 1991a; Schneider et al. 2017a) and phosphorus (Elliott et al. 1993; Schneider et al. 2017a) and nutrient deficiencies accelerate the rate of RCS by $12 \%$ compared to control conditions (Schneider et al. 2017a). The identification of additional treatments that accelerate or reduce RCS will be useful in studies evaluating the functional implications of RCS independent of tissue age as well as aid in understanding the genetic architecture of this phene.

\section{Analogies with leaf senescence}

Addicott and Lyon (1973) and later MacLeod et al. (1986) and Henry and Deacon (1981) proposed that RCS may be analogous to the senescence of leaves and shoots. RCS and leaf senescence have many similarities; they both are accelerated by deficiencies in mobile nutrients (e.g. nitrogen, phosphorus) (Tully et al. 2013; Schneider et al. 2017a), are accelerated by calcium deficiency, a known regulator of senescence (Ferguson 1984; Lascaris and Deacon 1991a), are influenced by ethylene (Jackson and Osborne 1970; Schneider et al. 2017c), accumulate pathogenesisrelated (PR) proteins in very early stages of senescence (Tamas et al. 1998; Quirino et al. 2000; Liljeroth et al. 2001), and are a type of PCD (van der Kop et al. 2003; Schneider et al. 2017c). Defender Against Death 1 (DAD1), a negative regulator of PCD (Moharikar et al. 2007), is significantly downregulated at the onset of RCS in barley roots (Schneider et al. 2017c), petal senescence of pea (Orzaez and Granell 1997a; Orzaez and Granell 1997b) and gladiolus (Yamada et al. 2004), and leaf senescence of iris and carnation (van der Kop et al. 2003). However, RCS and leaf senescence are different with respect to regulation and implications for plant fitness. Cycloheximide, a protein synthesis inhibitor that delays senescence in leaf disks (Martin and Thimann 1972), did not reduce the rate of RCS in wheat (Lascaris and Deacon 1991b). In addition, leaf senescence is accelerated by damage to cellular constituents caused by free radicals (Kellogg and Fridovich 1975; Strother 1988). However, sodium benzoate, gallic acid, and ascorbic acid, known free radical scavengers, had no effect on the rate of RCS in wheat (Lascaris and Deacon 1991b). In addition, leaf and root cortical senescence have fundamentally different biological functions.
RCS involves senescence of a specific tissue type within an organ, while leaf senescence culminates in abscission of an entire organ. However, in leaves of some species of Monstera and related genera, the formation of a complex leaf shape involves the PCD in regions of leaf tissue, rather than a specific tissue type or organ abscission (Gunawardena 2007). In monocarpic senescence of deciduous plants, leaves senesce simultaneously as triggered by genetic and environmental cues (e.g. temperature, photoperiod) (Tully et al. 2013). In annual plants, during senescence resources in the leaves are translocated to developing seeds and fruits. In perennials, these resources may be stored in stems or roots and may be used in the following season (Tully et al. 2013). In contrast, RCS begins in root cortical tissue as early as a few weeks after germination and senescence progresses throughout plant growth, and only the rate may be triggered by environmental cues (Schneider et al. 2017a). RCS may be an adaptive strategy to improve plant growth in edaphic stress conditions. Leaf senescence and the development of RCS have similarities with respect to the modulation (i.e. nutrient deficiencies, ethylene) of the PCD process. However, RCS and leaf senescence are fundamentally different in respect to adaptation and fitness.

\section{RCS and source-sink relations}

As an alternative to the leaf senescence theory, Henry and Deacon (1981) proposed a sink-strength model to explain the development of RCS. In this model, RCS may result from reduced or inadequate supply of photoassimilates reaching the root cortex from the stele and the cortex not acting as an efficient resource sink compared to the growing axial and lateral root tips (Henry and Deacon 1981). The pattern of RCS matches this theory; a reduction in photosynthate from the stele would result in a reduction of resources reaching the epidermis and outer cortical layers first, where RCS begins. However, we would also expect the rate of RCS to increase as environmental conditions reduce the allocation of photoassimilates to the roots. In wheat, leaf pruning did not affect the rate of RCS (Lascaris and Deacon 1991a). In Poa pratensis, increased temperature increased the rate of RCS (Smiley and Giblin 1986). However, shading decreased the rate of RCS in wheat and barley (Lewis and Deacon 1982) and there was no clear effect of shading on RCS development in Poa pratensis (Smiley and Giblin 1986). 
Similar to the sink-strength model, Henry and Deacon (1981) also proposed that endodermal suberization and cell wall thickening restrict the movement of photoassimilates into the root cortex, thereby inducing RCS. RCS was not correlated with the age or distance of lignification in the Casparian strip, tertiary endodermis, protoxylem, metaxylem, or stellar ground tissues as assessed by phloroglucinol-HCl staining (Henry and Deacon 1981). Generally, in wheat and barley, lignification of the Casparian strip occurred before the onset of RCS and the lignification of the tertiary endodermis occurred after the senescence at least four cortical cell files (Henry and Deacon 1981). However, RCS coincided with increased aliphatic suberin in the endodermis and these differences were more pronounced in landraces, which had a significantly faster progression of RCS compared to modern varieties (Schneider et al. 2017a). Staining methods detecting lignin and GC-MS methods detecting lipids, cannot discern if RCS is the cause or effect of endodermal development or if they are indirectly related by unknown mechanisms. Suberization of the endodermis may restrict the movement of assimilates to the cortex, starving cortical cells, inducing senescence as Henry and Deacon (1981) proposed. However, we propose that increased endodermal suberization may occur after the development of RCS in order to protect the root from pathogen invasion and radial water and nutrient loss after the senescence of cortical and epidermal cells.

\section{Functional implications of RCS}

RCS and metabolic costs

RCS reduces the carbon and nutrient costs of soil exploration by destroying living cortical tissue, thereby reducing carbon and nutrient costs of maintaining a living cortex (Schneider et al. 2017a). After the development of RCS, assimilates that would have been partitioned to the root for maintenance of the cortex may be used for the growth of shoots or new roots, which can increase soil exploration (Fig. 3). Per unit root length, nitrogen and phosphorus content were reduced by $80 \%$ in root segments with complete cortical senescence compared to root segments with no RCS at 35 days of growth (Schneider et al. 2017a). RCS reduced root respiration by $87 \%$ per unit dry weight and $96 \%$ per unit length compared to root segments with no

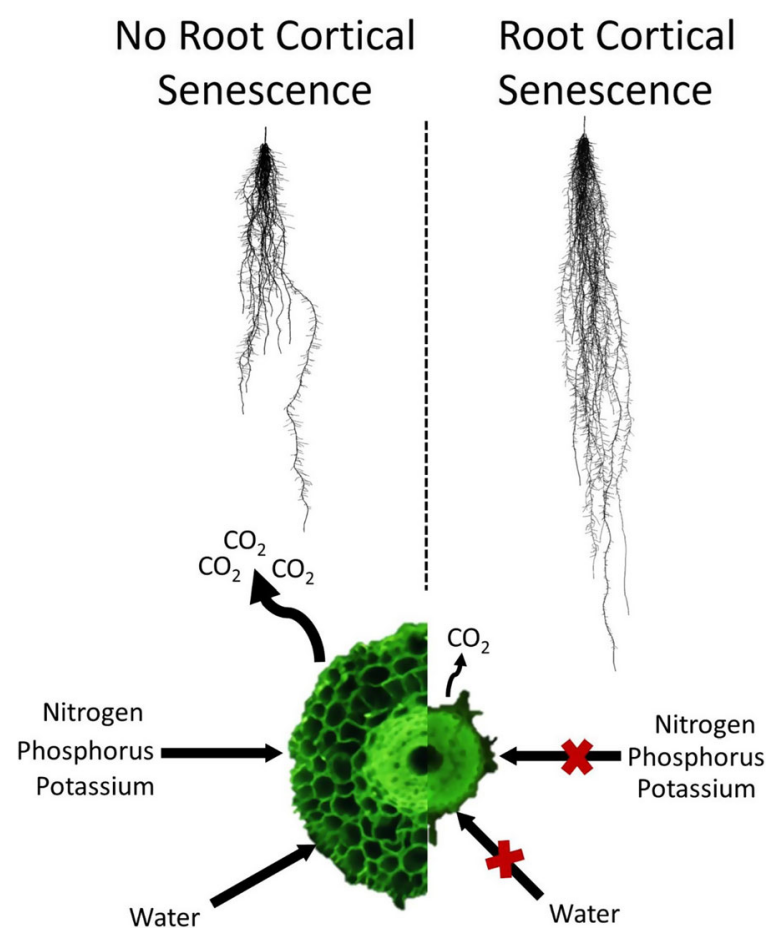

Fig. 3 Schematic of RCS and soil resource capture. In edaphic stress, plants with RCS have greater root length, reduced root respiration, and reduced radial water and nutrient uptake. Greater root growth in plants with RCS is driven by savings in metabolic costs of root tissue. Reduced radial water and nutrient transport of axial root tissue after RCS has small effects on total plant nutrient uptake, as lateral roots do not form RCS and perform the majority of root nutrient and water uptake

RCS at 35 days of growth (Schneider et al. 2017a). SimRoot, a functional-structural plant model, demonstrated that reduced respiration of the cortex after RCS had relatively small effects on plant growth up to 80 days of growth, but large effects on plant carbon reserves. Plants with RCS had 54\% more non-structural carbon reserves compared to plants with no RCS at 80 days of growth. Under suboptimal nutrient availability, plants with RCS had significantly greater root length and significantly reduced respiration per root length compared to plants with no RCS (Schneider et al. 2017b).

RCS and radial transport of water and nutrients

The progression of RCS is correlated with progressively reduced radial hydraulic conductivity and radial transport of nitrogen and phosphorus. In excised barley root segments, RCS reduced radial hydraulic conductivity by $90 \%$, radial nitrate transport by $92 \%$, and radial phosphorus transport by $84 \%$ (Schneider et al. 2017a). 
Simulation results demonstrated that at anthesis, reduced radial nutrient transport and hydraulic conductivity due to RCS development had only neutral or small negative effects on plant growth under suboptimal availability of nitrogen, phosphorus, and potassium. Reduced radial nutrient transport after the development of RCS only slightly reduced total nutrient uptake throughout plant growth in suboptimal and optimal nutrient availabilities (Schneider et al. 2017b). Radial nutrient and water transport as a result of RCS is only disturbed in axial roots, as RCS does not develop in lateral roots. Lateral roots comprise the majority of root length and perform the majority of nutrient uptake compared to seminal and nodal axes (Schneider et al. 2017b). The development of RCS in axial root tissue presumably has little effect on total plant nutrient uptake and plant growth as RCS develops in older root segments that occupy soil domains that have already been depleted of nutrients by the younger root tissue of the same root axes. We speculate that RCS not only affects the radial transport of nitrogen, phosphorus, and potassium, but many other nutrients including calcium and magnesium.

We also proposed that a reduction in radial hydraulic conductivity may not be a tradeoff to RCS, but rather a benefit (Fig. 3). Reduced radial hydraulic conductivity may be advantageous under water limited conditions by conserving soil water throughout the growth season, a strategy known as "water banking" (Feng et al. 2016). Reduced metaxylem vessel diameter conserves soil water throughout the growth season (Richards and Passioura 1989). Reduced hydraulic conductance may prevent desiccation of the root tip and the soil surrounding the root tip, which is important since dry soil has greater impedance. Reduced water capture may also create moderate shoot water stress, which increases shoot water use efficiency. We speculate that a reduction in radial water transport as a result of RCS is functionally advantageous, rather than functionally antagonistic to plant growth, however further studies are required to confirm this hypothesis.

Reduced radial transport of water and nutrients may be due to changes in the apoplastic and cell-to-cell pathways, reduced root-soil contact, and increased endodermal suberization after RCS. The development of RCS presumably disrupts the continuity of the apoplastic and the cell-to-cell pathway resulting in a longer path length and reduced cross-sectional area for radial transport across these pathways. Reduced rootsoil contact after the senescence of the cortex may also reduce radial hydraulic conductivity. Contact of roots with soil aggregates is an important driver of hydraulic conductivity at the root-soil interface (Carminati et al. 2012; Zarebanadkouki et al. 2014). In addition, changes in endodermal suberization coinciding with RCS may also contribute to the reduction in radial hydraulic conductivity and nutrient transport. Characterization of endodermal passage cells after RCS development will be important, as they will still be able to take up ions from the soil solution (Peterson and Enstone 1996). Further studies are needed to characterize cellular pathways of water transport, the impact of reduced root-soil contact, and endodermal suberization after RCS development.

\section{RCS and nutrient remobilization}

Reallocation of nutrients after RCS also has important functional implications for plant growth, particularly under edaphic stress. It was originally proposed that remobilization of phosphorus from senescing cells may be beneficial to plant growth (Robinson 1990). We proposed that remobilization of nitrogen and potassium may also be beneficial and we tested these hypothesis in silico with SimRoot. Simulation results demonstrated that remobilization of nutrients after RCS was always beneficial and increased plant growth by $33 \%$, $100 \%$, and $27 \%$ under suboptimal availability of nitrate, phosphorus, and potassium, respectively. At anthesis, reallocation of nutrients had the largest effects on plant growth compared to other functional implications of RCS including reduced root respiration and radial nutrient transport (Schneider et al. 2017b). However, the utility of nutrient reallocation, reduced respiration, and reduced radial nutrient transport was estimated using simulations. Validation of the functional implications of RCS and identification of additional benefits and tradeoffs would benefit from validation in the field.

Root cortical phenes are important for plant performance, particularly under edaphic stress (Zhu et al. 2010; Chimungu et al. 2014a; Chimungu et al. 2014b; Saengwilai et al. 2014; Schneider et al. 2017b). Root phenes that reduce root metabolic costs including RCS, RCA, cortical cell file number, and cortical cell size reduce carbon and nutrient costs for soil exploration (Chimungu et al. 2014a; Lynch et al. 2014; Saengwilai et al. 2014; Chimungu et al. 2014b; Schneider et al. 2017a). RCS is a promising breeding target for improved soil resource acquisition. 


\section{Similarities and differences between RCS and RCA}

RCS and RCA are functionally similar in many aspects. Both RCS and RCA are accelerated by nutrient deficiencies (Gillespie and Deacon 1988; Drew et al. 1989; Elliott et al. 1993), reduce radial nutrient transport (Hu et al. 2014; Schneider et al. 2017a), reduce radial hydraulic conductivity (Fan et al. 2007; Schneider et al. 2017a), reduce metabolic costs (Zhu et al. 2010; Postma and Lynch 2011b; Jaramillo et al. 2013; Saengwilai et al. 2014; Chimungu et al. 2015), are influenced by exposure to ethylene (Lascaris and Deacon 1991b; Lenochová et al. 2009; Schneider et al. 2017c), and are types of PCD (Liljeroth and Bryngelsson 2001; Jiang et al. 2010; Schneider et al. 2017c). Two ethylene-related genes were upregulated during both RCS and RCA formation (Rajhi et al. 2011; Schneider et al. 2017c). Both RCS and RCA improve nutrient acquisition, driven by reduced root metabolic costs, and increase plant growth in edaphic stress (Zhu et al. 2010; Postma and Lynch 2011a; Postma and Lynch 2011b; Schneider et al. 2017b). However, reductions in nutrient and water transport and root respiration associated with RCS are significantly greater compared to RCA formation (Fan et al. 2007; Zhu et al. 2010; Postma and Lynch 2011b; Jaramillo et al. 2013; Hu et al. 2014; Saengwilai et al. 2014; Chimungu et al. 2015; Schneider et al. 2017a).

Despite the similarities, RCS and RCA have some functional differences and are anatomically distinct. RCA has been primarily studied as a response to hypoxic conditions and improves oxygen transport to the root tip and rhizosphere (He et al. 1996; Evans 2004; Thomas et al. 2005) which has been shown to be particularly important in submergence tolerance in rice (Ito et al. 1999). Presumably, RCS which culminates in a non-continuous epidermis, has no utility in hypoxic conditions. In addition, the formation of RCA begins in middle cortical cell files and progresses in a radial pattern leaving spokes of cortical tissue connecting outer cortical cell files with inner cortical cell files (Arikado 1955; Thomson et al. 1990; Kawai et al. 1998; Lenochová et al. 2009), in contrast to RCS which begins in outer cortical cell files and progresses inward towards the endodermis until the entire cortex has senesced (Holden 1975; Henry and Deacon 1981). The development of RCS is constitutive and is developed primarily in small temperate grains in the Poaceae family (Deacon and Mitchell 1985; Yeates and Parker 1985; Jupp and
Newman 1987; Liljeroth 1995). Species that develop RCS may also form RCA in specific environmental conditions (e.g. hypoxia) (Deacon and Mitchell 1985; Setter and Waters 2003). The development of RCA is a common phenomenon in angiosperms and forms in many more species when compared to RCS including maize and rice. RCS and RCA are both functionally, physiologically, and genetically similar and both largely involve standard PCD in the root cortex. Further studies are needed to define genetic and physiological differences between RCS and RCA.

\section{Synergisms with other plant Phenes}

RCS has synergistic interactions with other phenes for plant growth. The number of tillers has implications for the utility of RCS in edaphic stress conditions. In suboptimal nitrogen, phosphorus, and potassium availability, simulations demonstrated plants with RCS and few tillers had $15 \%, 23 \%$, and $32 \%$ greater shoot biomass, respectively compared to the expected additive effects (Schneider et al. 2017b). RCS had greater benefit in plants with fewer tillers. Increased tiller number in suboptimal fertility depleted limited soil nutrient resources faster and soil nutrient resources became limiting in later growth stages therefore reducing plant growth. Barley, wheat, and other species that form RCS can vary greatly in the number of tillers formed, particularly between landraces and modern varieties (Schneider et al. 2017c) which can have large implications for the utility of RCS (Schneider et al. 2017b).

Synergistic interactions also were identified between RCS and lateral branching densities. In low nitrogen conditions, simulation studies demonstrated plants with sparse lateral branching and RCS had 14\% greater shoot biomass compared to the expected additive effects. In low phosphorus conditions, plants with RCS and dense lateral branching had $14 \%$ greater shoot biomass compared to the expected additive effects. In low potassium conditions, plants with RCS and dense lateral branching had 5\% greater shoot biomass compared to the expected additive effects (Schneider et al. 2017b). RCS results in greater resource availability (i.e. greater carbon and nutrient availability due to reallocation after cell senescence), and allows the plant to grow more root length and creates the potential for a greater lateral branching density and/or length. Dense lateral branching has a greater utility for the capture of immobile nutrients, 
including potassium and phosphorus, compared to nitrogen (Postma et al. 2014). In contrast, decreased lateral branching density in suboptimal nitrogen availability decreased intra- and inter-root competition and had greater utility in plants with RCS. Sparse lateral branching has also been shown to have greater utility for nitrogen capture in maize (Zhan and Lynch 2015).

It has been hypothesized that RCS may have other synergistic interactions with root and plant phenes, particularly phenes that are beneficial for soil and resource capture, but have a high metabolic cost. For example, the number of nodal roots, a root phene with a high metabolic cost, interacts with RCA in maize, a phene that reduces the metabolic costs of the root to increase plant growth (York et al. 2013). RCS may interact with growth angle or other phenes that affect the placement of roots in different soil domains. In some nitrogen regimes, a steep growth angle increases nitrogen capture (Trachsel et al. 2013; Dathe et al. 2016) and its interaction with RCS may enable the deep placement of roots in soil to capture mobile nutrients. Phene synergisms are important drivers in evolution and plant adaptation and have strong interactions with environmental conditions.

\section{Quantifying RCS}

The progression of RCS has been documented in the greenhouse, solution culture, and in the field using a variety of methods. Histology techniques using cell viability stains have been the most common phenotyping method for RCS. Neutral red (Beckel 1956), Feulgen reagent (Holden 1975), trypan blue (Brown and Hornby 1987), acridine orange (Henry and Deacon 1981), and we presume many other cell viability stains are viable methods to assess RCS. Wenzel and McCully (1991) question the validity of acridine orange and cell viability staining methods to assess RCS. However, the development of RCS assessed by acridine orange staining highly correlated with assessment of RCS using Feulgen staining (Henry and Deacon 1981) and single cell pressure probe methods (Bingham 2007). Single cell turgor pressure measurements, acridine orange staining, TUNEL-assay, and Nomarski optics methods demonstrated the same pattern and relative rate of RCS development between different plant species (Henry and Deacon 1981; Wenzel and McCully 1991; Liljeroth and Bryngelsson 2001; Bingham 2007). We propose that acridine orange and other cell viability stains are valid methods to assess RCS.

In addition to histological techniques, other methods have been used to phenotype RCS. DNA fragmentation, an indicator of PCD as assessed by TUNEL-positive nuclei, occurred in cortical cells of wheat and barley seminal roots and was used to phenotype RCS. (Henry and Deacon 1981; Liljeroth and Bryngelsson 2002; Deng et al. 2010). In addition, the use of a single-cell pressure probe enabled the quantification of RCS along a root (Bingham 2007). It has also been proposed that diameter of the axial root may be an indicator of RCS as a strong positive correlation was found between the percentage of turgid cells using single cell turgor pressure measurements and root diameter $\left(r^{2}=0.65\right)$ (Bingham 2007). Presumably many methods may be used to phenotype RCS however consideration of growing environment as well as phenotyping resources must be taken into account as phenotyping root anatomy can be time- and labor-intensive.

The challenge in phenotyping RCS is not the method selected to assess RCS, rather it is how and where RCS is quantified in the root segment. Several cell viability stains and quantification techniques have been used to quantify RCS. Deacon et al. assessed RCS in wheat and barley by counting the number of cortical cell files with at least one viable nucleus (and a presumed maximum of six cell files) (Deacon and Mitchell 1985; Deacon and Lewis 1986; Kirk and Deacon 1986; Lascaris and Deacon 1991a). However, the number of cortical cell files in barley roots can range from five to 14 (Schneider, unpublished). While this method provides a quick technique to quickly phenotype many root anatomical samples, it is difficult to get a detailed evaluation on the progression and pattern of RCS within a root segment. To evaluate the progression and pattern of RCS within a root cross-section Schneider et al. (2017b) proposed an alternative method. A microtome was used to cut cross-sections $60 \mu \mathrm{m}$ in thickness, which enabled the precise counting of viable nuclei in the entire cross section using a confocal microscope, rather than from a single focus field. The number of viable nuclei were counted in the inner, middle, and outer cortical cell regions and nuclei counts per cortical region were normalized based on the average cell length of that cortical region. By comparing nuclei counts in the outer, middle, and inner cortical regions the progression of RCS within the cortex can be assessed. Schneider et al. (2017b) also proposed a more 
rapid assessment of RCS to quantify the amount of cortex senesced, not the pattern of RCS. The difference in cross-sectional areas between the sample and the root apex can be used to quantify the percent cortex senesced (Schneider et al. 2017a). These methods are easily implemented using solution culture or greenhouse grown roots where the entire intact root system can be excavated. However, phenotyping RCS in field-grown plants is difficult as fragile cortical tissue is easily lost during excavation and washing, and relative differences in living cortical tissue are difficult to quantify due to the challenge of excavating entire roots. Schneider et al. (2017a) used a soil core method to quantify the progression of RCS in the field. Soil cores were taken directly over a plant which enabled the quantification of the volume of living cortical tissue per depth in the field.

\section{Research prospects}

RCS results in a shift from absorptive and resource capture functions of young root tissue to supportive, axial transport functions of mature root tissue. This concept is similar to the functional fine root orders proposed by McCormack et al. (2015) which separated fine roots into two pools: a short lived absorptive pool and a longer-lived transport pool. Absorptive roots are distinguished by their high surface area to volume ratio, high mycorrhizal colonization, high root hair density, less suberization, greater respiration, greater tissue nitrogen concentration, and an intact cortex. Transport roots are distinguished by the high stele to diameter ratio, mature xylem conduits, the loss of the root cortex, and high suberization and lignification (McCormack et al. 2015). We propose that these concepts not only apply to fine roots of perennial species but may be applicable to annual species, particularly those which develop RCS. However, in the case of RCS, absorptive and transport classes may be based on root regions, rather than entire roots since the growing root tip functions as an absorptive root while the older root tissue has transport functions.

Additional insights on the functional implications of RCS including its effects on aquaporin expression may enable further classification of absorptive and transport root tissue. In many tissues, water transport across cell membranes is mediated by aquaporins, small integral membrane proteins that localize in subcellular compartments. Evaluating the contribution of aquaporins to root water uptake is difficult, however derivatives of mercury which act though oxidation and binding to Cysteine residues may be used as aquaporin blockers (Maurel et al. 2008). Presumably after RCS has occurred, and cortical tissue is dead, aquaporin expression does not contribute to any radial water flow. Studies have shown that in response to environmental signals, roots have the ability to respond to environmental signals by altering water transport through changes in aquaporin-mediated membrane permeability (Javot and Maurel 2002). The expression of aquaporins is important to consider in radial water transport (Javot and Maurel 2002) as they theoretically could change to alter water uptake and membrane permeability in roots during RCS.

The impact of RCS on mycorrhizal symbiosis is also largely unknown. As RCS progresses, reduced cortical habitat may reduce mycorrhizal symbiosis. This merits investigation. Additional tradeoffs of RCS development have yet to be explored. For example, it is unclear how the loss of the cortex affects pathogen resistance or the ability of the root to sequester $\mathrm{Na}$ and $\mathrm{Cl}$ under salinity stress.

A challenge in studying RCS is understanding its utility and tradeoffs in the context of specific environments, management practices, and in different phenotypic backgrounds, i.e. the 'fitness landscape' (Lynch and Brown 2012). As for many root phenes, there are fitness tradeoffs to the development of RCS (Schneider et al. 2017a). However, the utility and fitness tradeoffs for RCS in many environments have yet to be investigated, including the implications of RCS in drought environments. As with RCA, reduced cortical cell file number, and increased cortical cell size, we speculate that RCS improves drought tolerance (Zhu et al. 2010; Chimungu et al. 2014a, b). We speculate that RCS may have implications in drought tolerance by conserving soil water throughout the growth season. We also speculate that the RCS is accelerated under drought.

A major bottleneck to the integration of RCS in breeding programs is the challenge of phenotyping a large number of plants. Excavation of root crowns can be performed in the field on a large scale (Trachsel et al. 2011), however, anatomical phenotyping through image analysis remains tedious. The phenotyping of RCS is not possible at the seedling stage, since it takes several weeks of plant growth for the phenotype and genetic differences to be visible. The identification of genetic markers controlling RCS would greatly facilitate their use in breeding programs (Lynch 2011; Henry et al. 2012; York et al. 2013). Advances in laser ablation tomography may enable the phenotyping of root anatomical traits on targeted root 
segments in a high-throughput manner (Chimungu et al. 2014a; Saengwilai et al. 2014; Chimungu et al. 2014b). However, since RCS changes temporally and spatially on a root system and varies by root class, the analysis of several root segments on the same root or threedimensional imaging may be necessary. The identification of genetic markers and genes controlling RCS would also enable the identification of further similarities or differences between RCS and RCA. Since RCS and RCA have some of the same functional implications and their development are modulated by many of the same factors (e.g. ethylene, nutrient deficiencies), it will be important to further characterize the physiological and genetic mechanisms controlling these traits. For example, the mechanisms required for barley roots to form aerenchyma in hypoxia conditions instead of RCS are largely unknown.

It will be important to further characterize RCS in other species and members of the Poaceae. Limited work has been performed on identifying species that form RCS. In barley, landraces have a faster progression of RCS compared to modern varieties. Historically, landraces are grown in low-input systems and we speculate that the development of RCS may be a useful adaptation for resource capture in these environments. Modern varieties, typically selected using modern agronomic management practices including irrigation and fertilization, develop RCS, albeit at a reduced rate compared to landraces. Further research may evaluate the progression of RCS in different species of temperate grasses and how these rates vary in different environmentally adapted varieties.

We propose that RCS may not be controlled by a sink-strength model or develop as a stress symptom, but rather is a positive adaptation improving plant fitness under edaphic stress (Schneider et al. 2017a). The challenge of understanding the genetics, physiology, and fitness landscape of RCS will require in silico approaches and expertise in plant biology, soil science, and microbiology. We propose that RCS merits further characterization and consideration as a phene for adaptation to edaphic stress.

\section{References}

Addicott F, Lyon J (1973) Physiological ecology of abscission, In: physiological ecology: a series of monographs, texts, and treatises. Academic Press, New York p.85-123

Arikado H (1955) Anatomical and ecological responses of barley and some forage crops to the flooding treatment. Bulletin Faculty Agriculture, Mie University Tsu Mie 11:1
Azeez JO, Chikoye D, Kamara AY, Menkir A, Adetunji MT (2005) Effect of drought and weed management on maize genotypes and the tensiometric soil water content of an eutric nitisol in south western Nigeria. Plant Soil 276:61-68

Beckel DKB (1956) Cortical disintegration in the roots of Bouteloua gracilis (H.B.K) Lag. New Phytol 55:183-190

Bingham IJ (2007) Quantifying the presence and absence of turgor for the spatial characterization of cortical senescence in roots of Triticum aestivum (Poacaea). Am J Bot 94:2054-2058

Brisson N, Gate P, Gouache D, Charmet G, Oury FX, Huard F (2010) Why are wheat yields stagnating in Europe? A comprehensive data analysis for France. F. Crop Res 119:201-212

Brown ME, Hornby D (1987) Effects of nitrate and ammonium on wheat roots in gnotobiotic culture: amino acids, cortical cell death and take-all caused by Gaeumannoyces graminis var. tritici. Soil Biol Biochem 19:567-573

Carminati A, Vetterlein D, Koebernick N, Blaser S, Weller U, Vogel HJ (2012) Do roots mind the gap? Plant Soil 367: 651-661

Chimungu J, Brown K, Lynch J (2014a) Large root cortical cell size improves drought tolerance in maize (Zea mays $L$ ). Plant Physiol 166:2166-2178

Chimungu J, Brown K, Lynch J (2014b) Reduced root cortical cell file number improves drought tolerance in maize. Plant Physiol 166:1943-1955

Chimungu JG, Maliro MFA, Nalivata PC, Kanyama-Phiri G, Brown KM, Lynch JP (2015) Utility of root cortical aerenchyma under water limited conditions in tropical maize (Zea mays L.). F. Crop Res 171:86-98

Dathe A, Postma J, Postma-Blaauw MB, Lynch J (2016) Impact of axial root growth angles on nitrogen acquisition in maize depends on environmental conditions. Ann Bot 118:401-414

Deacon J (1980) Ectotrophic growth by Phialophora radicicola var. graminicola and other parasites of cereal and grass roots. Trans Br Mycol Soc 75:158-160

Deacon J, Henry C (1978) Death of the ceral root cortex: its relevance to biological control of take-all. Ann Appl Biol 89:100

Deacon J, Henry C (1980) Age of wheat and barley roots and infection by Gaeumannomyces graminis var tritici. Soil Biol Biochem 12:113-118

Deacon J, Lewis SJ (1982) Natural senescence of the root cortex of spring wheat in relation to susceptibility to common root rot (Cochliobolus sativus) and growth of a free-living nitrogenfixing bacterium. Plant Soil 66:13-20

Deacon J, Lewis S (1986) Invasion of pieces of sterile wheat root by Gaeumannomyces graminis and Phialophora graminicola. Soil Biol Biochem 18:167-172

Deacon J, Mitchell R (1985) Comparison of rates of natural senescence of the root cortex of wheat (with and without mildew infection), barley, oats, and rye. Plant Soil 84:129-131

Deng XY, Li JW, Zhou ZQ, Fan HY (2010) Cell death in wheat roots induced by the powdery mildew fungus Blumeria graminis $f$. sp. tritici. Plant Soil 328:45-55

Drew MC, He CJ, Morgan PW (1989) Decreased ethylene biosynthesis, and induction of aerenchyma, by nitrogen- or phosphate-starvation in adventitious roots of Zea mays L. Plant Physiol 91:266-271

Elliott B, Robson A, Abbott L (1993) Effects of phosphate and nitrogen application on death of the root cortex in spring wheat. New Phytol 123:375-382 
Evans DE (2004) Aerenchyma formation. New Phytol 161:35-49

Fan M, Bai R, Zhao X, Zhang J (2007) Aerenchyma formed under phosphorus deficiency contributes to the reduced root hydraulic conductivity in maize roots. J Integr Plant Biol 49: 598-604

Feng W, Lindner H, Robbins NE, Dinneny JR (2016) Growing out of stress: the role of cell- and organ-scale growth control in plant water-stress responses. Plant Cell 28:1769-1782

Ferguson IB (1984) Calcium in plant senescence and fruit ripening. Plant Cell Environ 7:477-489

Gillespie I, Deacon J (1988) Effects of mineral nutrients on senescence of the cortex of wheat roots and root pieces. Soil Biol Biochem 20:525-531

Griffin GJ, Hale MG, Shay FJ (1975) Nature and quanitity of sloughed organic matter produced by roots of axenic peanut plants. Soil Biol Biochem 8:29-32

Gunawardena AH (2007) Programmed cell death and tissue remodelling in plants. J Exp Bot 59(3):445-451

He CJ, Morgan PW, Drew MC (1996) Transduction of an ethylene signal is required for cell death and lysis in the root cortex of maize during aerenchyma formation induced by hypoxia. Plant Physiol 112(2):463-472

Henrici M (1929) Structure of the cortex of grass roots in the more arid regions of South Africa. Union of South Africa Department of Agriculture. Sci Bull 85:3-12

Henry C, Deacon J (1981) Natural (non-pathogenic) death of the cortex of wheat and barley seminal roots, as evidenced by nuclear staining with acridine orange. Plant Soil 60:255-274

Henry A, Mabit L, Jaramillo RE, Cartagena Y, Lynch JP (2012) Land use effects on erosion and carbon storage of the Río Chimbo watershed, Ecuador. Plant Soil 367:477-491

Ho MD, Rosas JC, Brown KM, Lynch JP (2005) Root architectural tradeoffs for water and phosphorus acquisition. Funct Plant Biol 32:737-748

Holden J (1975) Use of nuclear staining to assess rates of cell death in cortices of cereal roots. Soil Biol Biochem 7:333-334

Holden J (1976) Infection of wheat seminal roots by varieties of Phialophora radicicola and Gaeumannomyces graminis. Soil Tillage Res 8:109-119

Hu B, Henry A, Brown KM, Lynch JP (2014) Root cortical aerenchyma inhibits radial nutrient transport in maize (Zea mays). Ann Bot 113:181-189

Ito O, Ella E, Kawano N (1999) Physiological basis of submergence tolerance in rainfed lowland rice ecosystem. Field Crop Res 64:75-90

Jackson MB, Osborne DJ (1970) Ethylene, the natural regulator of leaf abscission. Nature (London) 225:1019-1022

Jaramillo RE, Nord EA, Chimungu JG, Brown KM, Lynch JP (2013) Root cortical burden influences drought tolerance in maize. Ann Bot 112:429-437

Javot H, Maurel C (2002) The role of Aquaporins in root water uptake. Ann Bot 90:301-313

Jiang Z, Song X-F, Zhou Z-Q, Wang L-K, Li J-W, Deng X-Y, Fan H-Y (2010) Aerenchyma formation: programmed cell death in adventitious roots of winter wheat (Triticum aestivum) under waterlogging. Funct Plant Biol 37:748-755

Jupp A, Newman I (1987) Morphological and anatomical effects of severe drought on the roots of Lolium perenne L. New Phytol 105:393-402

Kawai M, Samarajeewa PK, Barrero RA, Nishiguchi M, Uchimiya H (1998) Cellular dissection of the degradation pattern of cortical cell death during aerenchyma formation of rice roots. Planta 204:277-287

Kellogg E, Fridovich I (1975) Superoxide, hydrogen peroxide, and singlet oxygen in lipid peroxidation by a xanthine oxidase system. J Biol Chem 250:8812-8817

Kirk J, Deacon J (1986) Early senescene of the root cortex of agricultural grasses and of wheat following root amputation or infectino by the take-all fungus. New Phytol 104:63-75

Krauss U, Deacon J (1994) Root turnover of groundnut (Arachis hypogaea L .) in soil tubes. Plant Soil 166:259-270

Lascaris D, Deacon J (1991a) Relationship between root cortical senescence and growth of wheat as influenced by mineral nutrition, Idriella bolleyi (Sprague) von Arx and pruning of leaves. New Phytol 118:391-396

Lascaris D, Deacon J (1991b) Comparison of methods to assess senescence of the cortex of wheat and tomato roots. Soil Biol Biochem 23:979-986

Lenochová Z, Soukup A, Votrubová O (2009) Aerenchyma formation in maize roots. Biol Plant 53:263-270

Lewis S, Deacon J (1982) Effects of shading and powdery mildew infection on senescence of the root cortex and coleoptile of wheat and barley seedlings, and implications for root- and foot-rot fungi. Plant Soil 69:401-411

Liljeroth E (1995) Comparisons of early root cortical senescence between barley cultivars, Triticum species and other cereals. New Phytol 130:495-501

Liljeroth E, Bryngelsson T (2001) DNA fragmentation in cereal roots indicative of programmed root cortical cell death. Physiol Plant 111:365-372

Liljeroth E, Bryngelsson T (2002) Earlier onset of DNA fragmentation in leaves of wheat compared to barley and rye. Hereditas 136:108-115

Liljeroth E, Santen K, Bryngelsson T (2001) PR protein accumulation in seminal roots of barley and wheat in response to fungal infection - the importance of cortex senescence. $J$ Phytopathol 149:447-456

Liueroth E, Franzon-Almgren I, Gunnarsson T (1996) Root colonization by bipolaris sorokiniana in different cereals and relations to lesion development and natural root cortical cell death. J Phytopathol 144:301-307

Lobell DB, Roberts MJ, Schlenker W, Braun N, Little BB, Rejesus RM, Hammer GL (2014) Greater sensitivity to drought accompanies maize yield increase in the U.S. Midwest. Science 344:516-519

Lynch JP (2007) Rhizoeconomics: the roots of shoot growth limitations. Hortscience 45:1107-1109

Lynch JP (2011) Root phenes for enhanced soil exploration and phosphorus acquisition: tools for future crops. Plant Physiol 156:1041-1049

Lynch J (2013) Steep, cheap and deep: an ideotype to optimize water and $\mathrm{N}$ acquisition by maize root systems. Ann Bot 112: $347-357$

Lynch JP (2015) Root phenes that reduce the metabolic costs of soil exploration: opportunities for 21 st century agriculture. Plant Cell Environ 38:1775-1784

Lynch JP, Brown KM (2012) New roots for agriculture: exploiting the root phenome. Philos Trans R Soc Ser B 367:1598-1604

Lynch JP, Ho MD (2005) Rhizoeconomics: carbon costs of phosphorus acquisition. Plant Soil 269:45-56 
Lynch JP, Wojciechowski T (2015) Opportunities and challenges in the subsoil: pathways to deeper rooted crops. J Exp Bot 66: 2199-2210

Lynch JP, Chimungu JG, Brown KM (2014) Root anatomical phenes associated with water acquisition from drying soil: targets for crop improvement. J Exp Bot 65:6155-6166

MacLeod W, Robson A, Abbott L (1986) Effects of phosphate supply and inoculation with a vesicular-arbuscular mycorrhizal fungus on the death of the root cortex of wheat, rape and subterranean clover. New Phytol 103:349-357

Manschadi AM, Christopher J, deVoil P, Hammer GL (2006) The role of root architectural traits in adaptation of wheat to waterlimited environments. Funct Plant Biol 33:823-837

Martin C, Thimann KV (1972) The role of protein synthesis in the senescence of leaves. Plant Physiol 49:64-71

Maurel C, Verdoucq L, Luu D-T, Santoni V (2008) Plant aquaporins: membrane channels with multiple integrated functions. Annu Rev Plant Biol 59:595-624

McCormack ML, Dickie IA, Eissenstat DM, Fahey TJ, Fernandez CW, Guo D, Helmisaari HS, Hobbie EA, Iversen CM, Jackson RB, Leppalammi-Kujansuu J (2015) Redefining fine roots improves understanding of below-ground contributions to terrestrial biosphere processes. New Phytol 207:505-518

Moharikar S, D'souza JS, Rao BJ (2007) A homologue of the defender against the apoptotic death gene (dad1) in UVexposed Chlamydomonas cells is downregulated with the onset of programmed cell death. J Biosci 32:261-270

Old K, Nicolson T (1975) Electron microscopical studies of the microflora of roots of sand dune grasses. New Phytol 74:51-58

Orzaez D, Granell A (1997a) The plant homologue of the defender against apoptotic death gene is down-regulated during senescence of flower petals. FEBS Lett 404:275-278

Orzaez D, Granell A (1997b) DNA fragmentation is regulated by ethylene during carpel senescene in Pisum sativum. Plant J 11:137-144

Peterson CA, Enstone DE (1996) Functions of passage cells in the endodermis and exodermis of roots. Physiol Plant 97:592-598

Pieruschka R, Poorter H (2012) Phenotyping plants: genes, phenes and machines. Funct Plant Biol 39:813-820

Postma JA, Lynch JP (2011a) Theoretical evidence for the functional benefit of root cortical aerenchyma in soils with low phosphorus availability. Ann Bot 107:829-841

Postma JA, Lynch JP (2011b) Root cortical aerenchyma enhances the growth of maize on soils with suboptimal availability of nitrogen, phosphorus, and potassium. Plant Physiol 156: 1190-1201

Postma JA, Dathe A, Lynch J (2014) The optimal lateral root branching density for maize depends on nitrogen and phosphorus availability. Plant Physiol 166:590-602

Quirino BF, Noh YS, Himelblau E, Amasino RM (2000) Molecular aspects of leaf senescence. Trends Plant Sci 5:278-282

Rajhi I, Yamauchi T, Takahashi H, Nishiuchi S, Shiono K, Watanabe R, Mliki A, Nagamura Y, Tsutsumi N, Nishizawa NK, Nakazono M (2011) Identification of genes expressed in maize root cortical cells during lysigenous aerenchyma formation using laser microdissection and microarray analyses. New Phytol 190:351-368

Richards RA, Passioura JB (1989) A breeding program to reduce the diameter of the major xylem vessel in the seminal roots of wheat and its effect on grain yield in rain-fed environments. Aust J Agric Res 40:943-950
Robinson D (1990) Phosphorus availability and cortical senescence in cereal roots. J Theor Biol 145:257-265

Saengwilai P, Nord EA, Chimungu JG, Brown KM, Lynch JP (2014) Root cortical aerenchyma enhances nitrogen acquisition from low-nitrogen soils in maize. Plant Physiol 166:726-735

Schneider H, Wojciechowski T, Postma JA, Brown KM, Lücke A, Zeisler V, Schreiber L, Lynch JP (2017a) Root cortical senescence decreases root respiration, nutrient content, and radial water and nutrient transport in barley. Plant, Cell Environ 40:1392-1408

Schneider HM, Postma JA, Wojciechowski T, Kuppe C, Lynch J (2017b) Root cortical senescence improves barley growth under suboptimal availability of N, P, and K. Plant Physiol. pp. 00648

Schneider HM, Wojciechowski T, Postma JA, Brown K, Lynch J (2017c) Ethylene mediates root cortical senescence formation in barley. Dissertation, The Pennslyvania State University, University Park

Setter TL, Waters I (2003) Review of prospects for germplasm improvement for waterlogging tolerance in wheat, barley and oats. Plant Soil 253:1-34

Smiley R, Giblin D (1986) Root cortical death in relation to infection of kentucky bluegrass by Phialophora graminicola. Phytopathology 76:917-922

Sparks, Erin (2017): Arabidopsis root anatomy. Figshare doi: https://doi.org/10.6084/m9.figshare.4688344.v1

Strother S (1988) The role of free radicals in leaf senescence. Gerontology 34:151-156

Tamas L, Ciamporova M, Luxova M (1998) Accumulation of pathogenesis-related proteins in barley leaf intercellular spaces during leaf senescence. Biol Plant 41:451-460

Tebaldi C, Lobell DB (2008) Towards probabilistic projections of climate change impacts on global crop yields. Geophys Res Lett 35:L08705. https://doi.org/10.1029/2008GLO33423

Thomas AL, Guerreiro SMC, Sodek L (2005) Aerenchyma formation and recovery from hypoxia of the flooded root system of nodulated soybean. Ann Bot 96(7):1191-1198

Thomson CJ, Armstrong W, Waters I, Greenway H (1990) Aerenchyma formation and associated oxygen movement in seminal and nodal roots of wheat. Plant Cell Environ 13:395-403

Tilman D, Cassman KG, Matson PA, Naylor R, Polasky S (2002) Agricultural sustainability and intensive production practices. Nature 418:671-677

Trachsel S, Kaeppler SM, Brown KM, Lynch JP (2011) Shovelomics: high throughput phenotyping of maize (Zea mays L.) root architecture in the field. Plant Soil 341:75-87

Trachsel S, Kaeppler SM, Brown KM, Lynch JP (2013) Maize root growth angles become steeper under low $\mathrm{N}$ conditions. F. Crop Res 140:18-31

Tully KL, Wood TE, Schwantes AM, Lawrence D (2013) Soil nutrient availability and reproductive effort drive patterns in nutrient resorption in Pentaclethra Macroloba. Ecology 94:930-940

van der Kop DAM, Ruys G, Dees D, Van Der Schoot C, de Boer D, van Doorn WG (2003) Expression of defender against apoptotic death (DAD-1) in iris and Dianthus petals. Physiol Plant 117:256-263

Van Vuurde J, Schippers B (1980) Bacterial colonization of seminal wheat roots. Soil Biol Biochem 12:559-565 
Wenzel CL, McCully ME (1991) Early senescence of cortical cells in the roots of cereals. How good is the evidence? Am J Bot 78:1528-1541

Woods J, Williams A, Hughes JK, Black M, Murphy R (2010) Energy and the food system. Philos Trans R Soc B Biol Sci 365:2991-3006

Yamada T, Takatsu Y, Kasumi M, Marubashi W, Ichimura K (2004) A homolog of the defender against apoptotic death gene $(D A D 1)$ in senescing gladiolus petals is down-regulated prior to the onset of programmed cell death. J Plant Physiol 161:1281-1283

Yeates JS, Parker CA (1985) Rate of natural senescence of seminal root cortical cells of wheat, barley and oats, with reference to invasion by Gaeumannomyces graminis. Trans Br Mycol Soc 86:683-685

York LM, Nord EA, Lynch JP (2013) Integration of root phenes for soil resource acquisition. Front Plant Sci 4:355
Yu YB, Yang SF (1979) Auxin-induced ethylene production and its inhibition by aminoethyoxyvinylglycine and cobalt ion. Plant Physiol 64:1074-1077

Zarebanadkouki M, Kroener E, Kaestner A, Carminati A (2014) Visualization of root water uptake: quantification of deuterated water transport in roots using neutron radiography and numerical modeling. Plant Physiol 166:487-499

Zhan A, Lynch J (2015) Reduced frequency of lateral root branching improves $\mathrm{N}$ capture from low $\mathrm{N}$ soils in maize. $\mathrm{J}$ Exp Bot 66:2055-2065

Zhu J, Kaeppler SM, Lynch JP (2005) Topsoil foraging and phosphorus acquisition efficiency in maize (Zea mays). Funct Plant Biol 32:749-762

Zhu J, Brown KM, Lynch JP (2010) Root cortical aerenchyma improves the drought tolerance of maize (Zea mays L.) Plant Cell Environ 33:740-749 